\title{
Analysis of Learning Rate Using CPN Algorithm for Hand Written Character Recognition Application
}

\author{
W. H. Bangyal, J. Ahmad, and Q. Abbas
}

\begin{abstract}
This paper presents the analysis of the learning rate using the Counter Propagation network (CPN) algorithm for the hand written characters recognition application. The recognition process uses the forward only CPN algorithm to recognize the hand written characters. The experimental results obtained with different learning rate values shows that learning rate has large effect on the recognition process. Upper-case English alphabets for a number of different styles gathered from different peoples are used in the analysis for the performance of the CPN algorithm. The obtained recognition rates were $60 \%$ to $98 \%$ using the CPN for different learning rate value. The experimental results are very encouraging and satisfactory.
\end{abstract}

Index Terms-Handwritten character recognition, counter propagation networks, learning rate, performance.

\section{INTRODUCTION}

ANN can be more impressive as computational processors for different tasks like data compression, classification, combinatorial optimization problem solving, pattern recognition etc.

ANN has many advantages over the other classical methods. While having the computational complexity, ANN offered many advantages in pattern recognition adapting a very little context of human intelligence [11].

Communication is an integral component of human society that has been greatly enhanced by the evolution of the technological age. Verbal communication has been revolutionized by the advent of television, telephone and radio technology, and written communication has likewise been transformed by the development of word processors and electronic data. Handwriting is a natural means of communication which nearly everyone learns at an early age. Thus it provides a means of data entry for computers in which the user needs virtually no training. Handwriting recognition can be approached from both perspectives, and the current focus of the market today is on-line handwriting recognition. This is due to the popularity of personal management devices which involve the use of digital pen architecture

In the future, handwriting may only thrive more because of the technological developments under way that intend to establish handwriting as a new mode for humans to communicate with computers [9]. Handwriting has long been

Manuscript received October 19, 2012; revised January 12, 2013.

W. H.Bangyal is with the Department of Computing and Technology Iqra University Islamabad, Pakitsan (e-mail: waqas_bangyal@hotmail.com)

J. Ahmad is with Abasyn University Peshawar, Pakistan (e-mail: jamil.ahmad@abasyn. edu.pk).

Q. Abbas is with the Department of Computing and Technology Iqra University Islamabad, Pakistan (e-mail: qamar.bhk@gmail.com). studied by different fields for various different aspects and purposes, and it includes neuroscience, engineering, computer science, anthropology, education, etc [1]-[3].

Hand writing recognition has become very popular field in the last few years Handwriting recognition can be divided into two categories: on-line recognition and off-line recognition. In on line recognition, the writing person is physically connected to a computer through a mouse, an electronic pen, and the style of handwriting is recorded as a time dependent process. While in off line systems the data is collected on static image as the writing process is finished

Pattern recognition is the research area that is used to study and design of the system for the recognition of the different pattern either it is in the form of text, image or numeric [8].

In the last few decades, many applications have been developed to solve the different problems like as such as face recognition [4] and character recognition [5]. From the last few decades, a lot of research is carrying on in the field of handwriting recognition [6].

The paper presents the analysis of handwritten character recognition using the forward only $\mathrm{CPN}$ algorithm. Twenty six upper case English alphabets are considered in this paper for the experiments. No restriction is applied on the style of writing. This learning rate parameter of the neural network has a great affect on the accuracy of the network results.

In this paper forward only counter propagation network is used, and it is combination of unsupervised and supervised learning together proposed by Robert Hecht- Nielson[7], consisting of input layer, competitive layer and output layer. From input layer to competitive layer form the Kohonen network, while from competitive layer to and output layer form the Gross berg network. Using the Winner- Take-All strategy in the competitive learning, one neuron can be winner in the competitive layer and set its status to 1 while other to 0 .

The rest of the paper is organized as follows. In Section 2, background and related work are introduced. The Section 3 gives an overview of the proposed system. Section 4 describes the feature extraction steps from the handwritten character. In Section 5 the experimental results are provided with some analyses and discussions. Lastly Section 6 presents the concluding remarks and future work.

\section{LITERATURE SURVEY}

This paper present an analysis of the learning rate of forward only $\mathrm{CPN}$ for the hand written character recognition application. In an effort to determine the applicability of the forward only CPN for the problem of the hand written character recognition application, an analysis is conducted using the learning rate value. 
As learning rate is used in ANN more frequently but still not as much analyzed for any application specially pattern recognition applications. Learning rate plays an effective important rule in any application.

In [8] the author used Kohonen Neural Network for the license plate number identification character recognition application and its performance for the license plate number identification and the result shows that it gives the better performance with $78.57 \%$ of accuracy in recognizing the particular plate number.

In [9] the author used CPN Neural Network for the implementation of the remembering the user of the telephone number from a given list, even if the user only remembers part of a number or if the given number list having a series of exchanged digits. The input consists of from 0 to 9 digits and processing is performed on input number and it returns the selected number among all the learned number. It gives a simple learning methodology by assigning a number to the weights and in that way answer is obtained in a quick way.

In [10] the author used CPN Neural Network for the improvement of the accuracy of the grade of bank note recognition. The results show that $\mathrm{CPN}$ can solved the bank note recognition problem and speed of $\mathrm{CPN}$ is much better than the BP

In [10] the author used CPN Neural Network for on line hand written recognition application and the results the recognition rates were $60 \%$ to $94 \%$ using the $\mathrm{CPN}$ for different sets of character samples.

In [14] the author have used Neural Network classifier as a statistical methodology for feature extraction of hand written digits and obtained the accuracy up to $98 \%$ in the results of the experiments.

In [11] the author have used CPN Neural Network for the on line character recognition classifier and described the feature extraction methodology in detail and the results of the accuracy were from $60 \%$ to $90 \%$ in the application

\section{The Proposed Network ArChitecture}

I have proposed forward only counter propagation network for the analysis of the hand written character recognition application. The forward only CPN network has three layers. First layer is called the input layer. The second layer is known as kohonen (competitive layer) and third layer is called as Gross berg layer.

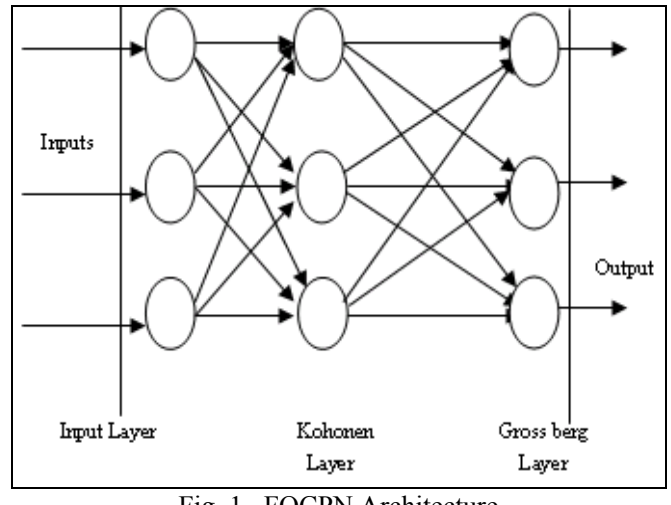

Fig. 1. FOCPN Architecture

The characters that are being used here are English alphabets written in the form of a matrix of $10 \times 10$. The parameters can affect the forward only counter propagation network k algorithm in term of the accuracy of result. Several experiments have been performed to look into the effect of using different learning rate. The analysis for the recognition of the hand written character is now done on the basis of different learning rate value that shall be discussed next.

\section{LEARNING/TRAINING}

In case study, 26 English Upper case alphabets characters have been used. Each writer was asked to write on writing area. No restriction was imposed on the content or style of writing. The writers consisted of university students, professors, and employees in the university.

The character is digitized into $10 \times 10=100$ digital cells, each having a single color, either black or white. It is important to encode this information into a useful meaningful form to a computer. For this, assign a value +1 to each black pixel and 0 to each white pixel and create the binary image matrix. The size of the matrix is defined by trial and error method in order to find the best matrix size of $10 \times 10$.

A $10 \times 10$ grid results in 100 inputs vectors and the boxes found with character pixels are considered "as on" and the remaining considered as "off" then it will presented to the neural network input for training and recognition purposes. The pre-processing of these hands written English alphabets characters is to convert them in to two dimensional matrixes of binary 0 and 1 . The binary 1 show the existence of the character in the cell and binary 0 shows that that there is no character in the cell. A file is maintained of these 1000 hand written upper case English alphabets characters and then used in simulation to get the results. Now this matrix containing binary 0 and 1 , to be used as Input for training and testing of the ANN.

And for training I have collected data of about 10000 characters 40 sample of each of the twenty six characters is used to get the results. For effective training of the designed network (to avoid over-fitting), the testing sets of 250 characters are used randomly.A set of examples used only to assess the performance or generalization of a fully-specified classifier [12] [13].

\section{EXPERIMENTAL RESULTS}

As it is described earlier that testing is performed on the basis of the samples taken from individuals who did not participate in the initial process of setting up the training data sets. The accuracy of results for hand written characters shown in the Fig. 2.

TABLE I: ACCURACY OF RESUlts AgAINST DifFERENT LEARNING RATE

\begin{tabular}{|c|c|c|c|c|c|}
\hline \multirow[b]{3}{*}{ Learning Rate } & \multicolumn{4}{|c|}{ VALUE } & \\
\hline & \multicolumn{5}{|c|}{ Recognition Rate \% } \\
\hline & NO Noise & $\begin{array}{c}5 \% \\
\text { Noise } \\
\end{array}$ & $\begin{array}{l}10 \% \\
\text { Noise } \\
\end{array}$ & $\begin{array}{l}15 \% \\
\text { Noise } \\
\end{array}$ & $\begin{array}{l}20 \% \\
\text { Noise } \\
\end{array}$ \\
\hline 0.9 & 98 & 96 & 94 & 75 & 57 \\
\hline 0.8 & 92 & 90 & 88 & 60 & 40 \\
\hline 0.7 & 88 & 86 & 82 & 52 & 35 \\
\hline 0.6 & 86 & 80 & 78 & 50 & 31 \\
\hline 0.5 & 80 & 75 & 70 & 48 & 23 \\
\hline
\end{tabular}




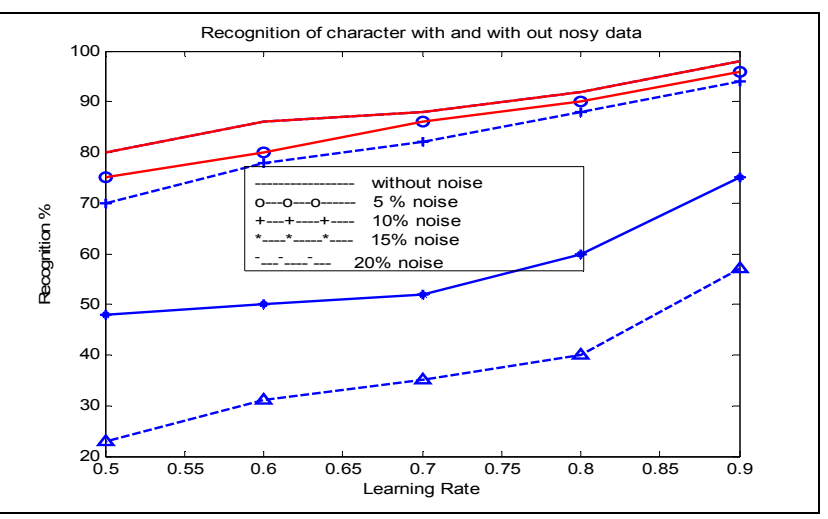

Fig. 2. Simulation result for the accuracy of results against learning rate

\section{A. Analysis}

From Table I, have the following observations and it shows that learning rate and recognition rate can be analyzed with and without noisy data as follows

- The value of accuracy increases as the learning rate increases as shown in corresponding table and graph. The value of accuracy decreases as the noise is increased in the data sets. Recognition rate is up to $98 \%$ So the learning rate and noise affects the performance of the proposed CPN.

- As noise increases in the data sets, value of accuracy decreases with the decrease in the learning rate.

\section{B. Analysis}

From Table II, have the following observations and it shows that learning rate, training samples and recognition rate can be analyzed as follows

- The value of accuracy increases as shown the training samples increases as in corresponding table and graph. Recognition rate is up to $98 \%$

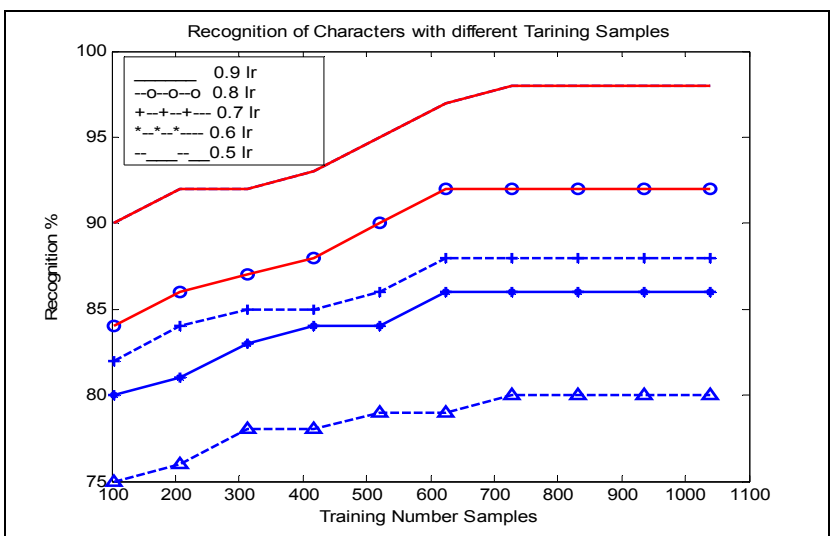

Fig. 3. Simulation result for the accuracy of results against learning rate with different training samples

TABLE II: TRAINING Time of Results Against DifFERENT LEARning RATE VALUE With DifFERENT TRAINING SAMPLES

\begin{tabular}{cccccccccc}
\hline \multicolumn{2}{c}{ Lr 0.9 } & \multicolumn{2}{c}{ Lr 0.8 } & \multicolumn{2}{c}{ Lr 0.7 } & \multicolumn{2}{c}{ Lr 0.6 } & \multicolumn{2}{c}{ Lr 0.5 } \\
\hline Samples & Accuracy & Samples & Accuracy & Samples & Accuracy & Samples & Accuracy & Samples & Accuracy \\
\hline 104 & 90 & 104 & 84 & 104 & 82 & 104 & 80 & 104 & 75 \\
\hline 208 & 92 & 208 & 86 & 208 & 84 & 208 & 81 & 208 & 76 \\
\hline 312 & 92 & 312 & 87 & 312 & 85 & 312 & 83 & 312 & 78 \\
\hline 416 & 93 & 416 & 88 & 416 & 85 & 416 & 84 & 416 & 78 \\
\hline 520 & 95 & 520 & 90 & 520 & 86 & 520 & 84 & 520 & 79 \\
\hline 624 & 97 & 624 & 92 & 624 & 88 & 624 & 86 & 624 & 79 \\
\hline 728 & 98 & 728 & 92 & 728 & 88 & 728 & 86 & 728 & 80 \\
\hline 832 & 98 & 832 & 92 & 832 & 88 & 832 & 86 & 832 & 80 \\
\hline 936 & 98 & 936 & 92 & 936 & 88 & 936 & 86 & 936 & 80 \\
\hline 1040 & 98 & 1040 & 92 & 1040 & 88 & 1040 & 86 & 1040 & 80 \\
\hline
\end{tabular}

\section{CONCLUSION}

The paper concludes that the proposed algorithm has very good capability of the hand written character recognition. And from the performance analysis it can be concluded that the learning rate affects the accuracy of the recognition of the hand written character recognition. And also it is observed that as the training samples increases accuracy also increases. So, the selection of learning rate plays a vital role in the recognition of hand written characters application and effect the accuracy of the results.

\section{REFERENCES}

[1] M. Simner, W. Hulstijn, and P. Girouard (Eds.), "Forensic, developmental and neuropsychological aspects of handwriting," Special issue of the Journal of Forensic Document Examination, 1994.

[2] R. Plamondon, Pattern Recognition, special issue on automatic signature verification, vol. 8, no. 3, June 1994
[3] G. P. Van Galen and P. Morasso, "Neuromotor control in handwriting and drawing," Acta Psychologica, vol. 100, no. 1-2, p. 236, 1998.

[4] G. Passalis, I. A. Kakadiaris, T. Theoharis, G. Toderici, and N. Murtuza "Three-Dimensional face recognition in the presence of facial expressions: An annotated deformable model approach," IEEE Transactions on Pattern Analysis and Machine Intelligence, vol. 29, no. 4, pp. 640-650, April 2007.

[5] L. Xiaobo, L. Xiaojing, and H. Wei. "Vehicle license plate character recognition," in Proc. IEEE Int. Conf. Neural Networks and Signal Processing, pp. 1066-1069, 2003.

[6] A. R. Khan and D. Muhammad, "A simple segmentation approach for unconstrained cursive handwritten words in conjunction with the neural network," International Journal of Image Processing, vol. 2, issue (3) 29, 2007

[7] H. Nielsen, "Counterpropagation networks," in Proc. IEEE First Int'l Conference on Neural Networks, 1987 II: 19-32.

[8] M. Yusoff, S. A. Rahman, S. Mutalib, and A. Mohamed, "Kohonen neural network performance in license plate number identification", in Proc. International Conference on Electrical Engineering and Informatics Institut Teknologi Bandung, Indonesia, pp. 20-24, 2007.

[9] J. Moreno, G. Sebastián1, M. A. Fernandez, and A. F. Aballero, "A telephone number corrector using acounterpropagation network," in Proc. the Fifth International Conference on Neural Information Processing ICONIP'98, Kitakyushu, Japan, pp. 1-6,1998. 
[10] M. F. Zafar, D. Mohamad, and R. M. Othman, "On-line handwritten character recognition: An implementation of counterpropagation neural net," World Academy of Science, Engineering and Technology, pp. 1-6, 2007.

[11] H. Altun and G. Gelen, "Enhancing performance of MLP/RBF neural classifiers via an multivariate data distribution scheme," Nigde University, Electrical and Electronics Engineering Department Nigde, Turkey, pp. 1-6, 2002.

[12] S. Haykin, Neural Networks, A Comprehensive Foundation, Second edition, 2005.

[13] H. Cecotti and A. Belaid, 2005, "Rejection strategy for Convolutional Neural Network by adaptive topology applied to handwritten digits recognition," in Proc. IEEE International Conference on Document Analysis and Recognition (8) (2005) 765-769.

[14] E. O. Justice and R. A Sundayn, "An optimum solution for a process control problem (continuous stirred tank reactor) using a hybrid neural network," Journal of Theoretical and Applied Information Technology, vol. 4, no. 10. pp.1-10, 2008.

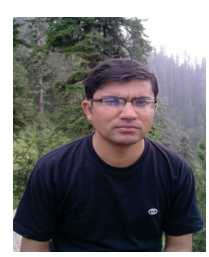

W. H. Bangyal received the BSc degree in mathematics and physics in 2001 from Govt, College Multan, Pakistan, MSc degree in Computer Science in 2003 from Bahauddin Zakariya University Multan, Pakistan, He completed his MS degree in computer science in 2009 from Iqra University Islamabad, Pakitsan. Currently he is a $\mathrm{PhD}$ (computer science) student at the Department of Computing and Technology Iqra University Islamabad, Pakistan. His research interests include pattern recognition, neural networks and evolutionary algorithms. He is an active member of several professional Societies. 